\title{
Pneumonien enden oft tödlich
}

\author{
Hohes Risiko im Alter Pflegebedürftige Personen sind besonders gefährdet \\ an einer Pneumonie zu erkranken. Alte und pflegebedürftige Menschen haben \\ zudem ein sehr hohes Risiko, daran zu versterben. Welche Anzeichen deuten \\ auf eine Pneumonie? Können Pflegende prophylaktisch gegen das Entstehen \\ einer Lungenentzündung vorgehen?
}

D as Risiko, an einer Pneumonie zu erkranken, steigt ab dem 60. Lebensjahr mit jedem Lebensjahrzehnt an. Bewohner von Pflegeheimen gelten als besonders gefährdete Gruppe. Mit etwa $16 \%$ aller Notrufe in Heimen sind Pneumonien der zweithäufigste Grund ärztlicher Einsätze. Neben dem Alter werden Funktionseinbußen in der Selbstversorgung, Schluckstörungen, Bettlägerigkeit und zusätzliche Erkrankungen als personenbezogene Risiken genannt. Zu den Erkrankungen mit Pneumonie-Risiko zählen insbesondere chronische Atemwegserkrankungen, Herzinsuffizienz und akute grippale Infekte. Verstärkt wird das Gesamtrisiko durch Untergewicht und Unterernährung, hohen Alkoholkonsum sowie aktives und passives Tabakrauchen. Weitere Risikofaktoren für die Ausbildung einer Pneumonie sind reduzierte Immunabwehr und mangelhafte Mundhygiene.

Für die Risikoeinschätzung liegen keine Instrumente vor, die als aussagegültig und zuverlässig gelten. Allen fehlt es an Objektivität, um sie empfehlen zu können. Deshalb ist die klinische Einschätzung der Pflegepersonen, die aus den beschriebenen Risikofaktoren zusammen mit der beruflichen Erfahrung resultiert, das Mittel der Wahl, um Risikopersonen zu identifizieren.

\section{Anzeichen einer Pneumonie}

Die im Pflegeheim erworbene Pneumonie wird mit dem englischen Diagnosetitel als „NHAP - nursing-home-acquired-pneumonia“ bezeichnet. Sie gilt grundsätzlich wegen des hohen Sterberisikos als Notfall, der einen umgehenden Arzteinsatz rechtfertigt. Etwa 10\% der Bewohner mit Pneumonie versterben daran innerhalb der ersten 78 Stunden nach Krankenhauseinweisung.Deshalb sind die Krankenbeobachtung und die Beschreibung erhobener Symptome an den Arzt von entscheidender Bedeutung. Äußere Zeichen einer Pneumonie sind:

- Atemwegssymptome wie Luftnot (Dyspnoe)

- Atemabhängige Schmerzen im Brustkorb

- Husten mit oder ohne Auswurf

- Rasselgeräusche beim Einatmen

- Kreislaufbeschwerden und Schwindelempfinden
_ „Grippale Symptome“ und allgemeines Krankheitsgefühl

- Fieber und Bewusstseinstrübung.

Im höheren Lebensalter treten Pneumonien häufig mit nur wenigen Symptomen oder sogar asymptomatisch auf. So können Fieber und Husten völlig fehlen. Dafür zeigen sich Verwirrtheit (delirantes Syndrom) und Diarrhoe, was zunächst in eine andere Richtung weisen kann. Deshalb muss die Prüfung der Atemfrequenz (unter 30/Min.) und des Blutdrucks (systolisch unter $90 \mathrm{mmHg}$, diastolisch unter $60 \mathrm{mmHg}$ ) immer vorgenommen werden.

\section{Dysphagie und Aspiration erhöhen das Risiko}

Ein auffallend hoher Anteil der Pneumonien im Alter ist auf Schluckstörungen zurückzuführen. Schluckstörungen treten bei alten Menschen zumeist altersphysiologisch, also auch ohne zugrundeliegende spezielle Erkrankung auf. Sie betreffen die reduzierte Wahrnehmung von Speiseresten im Mund, die eingeschränkte Kontrolle des Speisebreis und die verzögerte Auslösung des Schluckreflexes. Zudem kommt es altersbedingt zu einer verminderten Zungenbeweglichkeit, um Speisereste zu fassen und zu entfernen. Im höheren Alter findet sich zudem eine reduzierte Speichelproduktion, um das Milieu der Mundflora aufrecht zu erhalten. Hinzu kommen die teilweise fehlende Reinigung im Rachen durch Nachschlucken von Speichel und die eingeschränkte Öffnung des Ösophagusmuskels zur Sicherheit des Schluckaktes. Bei pflegebedürftigen Personen führen zusätzlich eine mangelhafte Mundhygiene und ein schlechter Zahnstatus zur Ansammlung von Bakterien im Mund-Rachen-Raum, sodass bei einer Aspiration größere Mengen von pathogenen Erregern in die Atemwege gelangen. Sobald die Möglichkeit, Schluckprobleme zu kompensieren, nicht mehr ausreicht, nimmt das Risiko einer Pneumonie zu. Zusätzlich ist in der Regel das Abhusten erschwert, wodurch die Keime im Atmungstrakt verbleiben.

Auch oft verordnete Medikamente wie Neuroleptika, Sedativa oder Hypnotika beeinträchtigen die Koordination zwischen Atmungs- und Schluckfunktion. Teilweise wird der Schluckreflex völlig herabgesetzt. Hierdurch gelangen Speichel und Speisen in die 


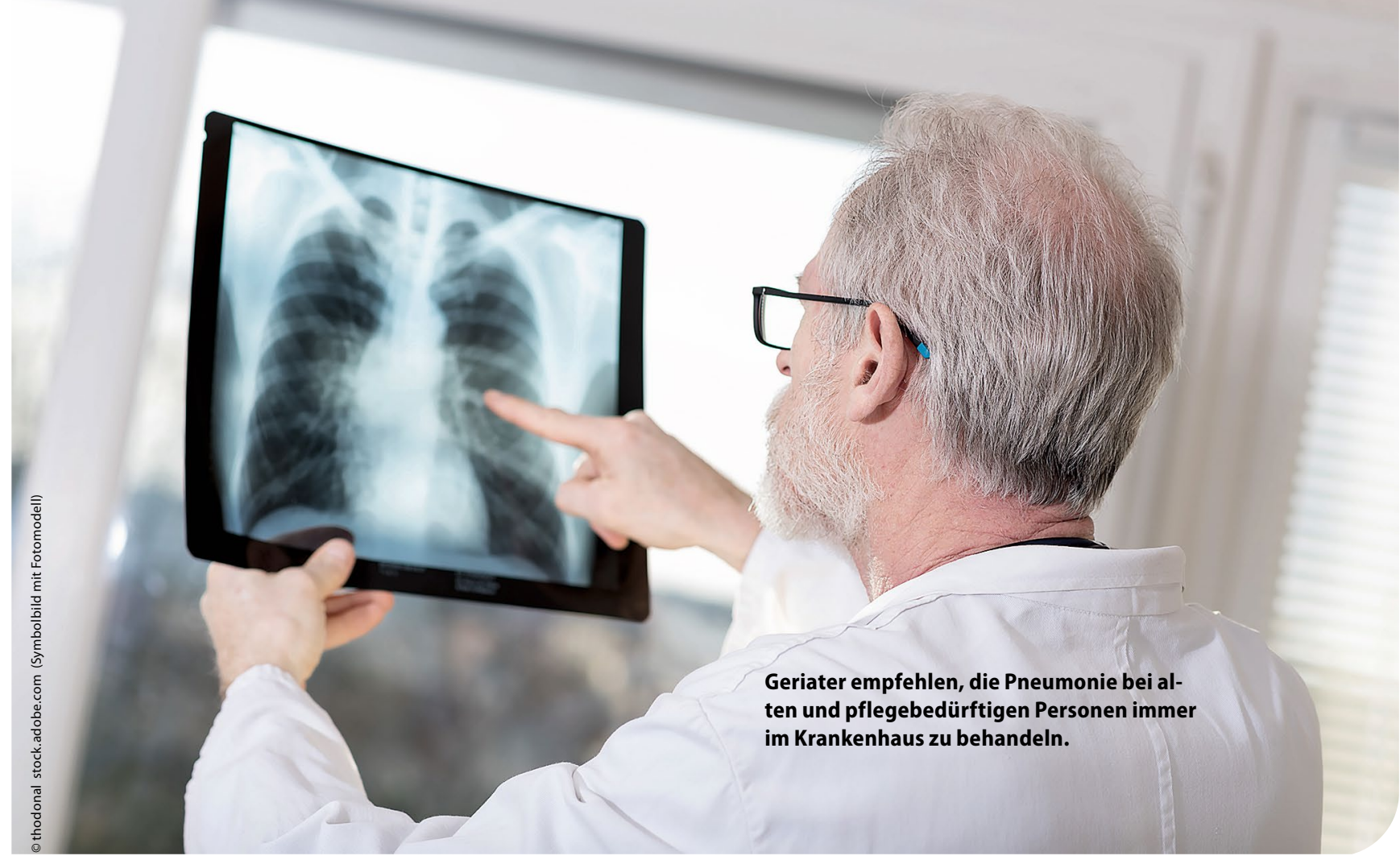

Luftröhre. Eine frühzeitige ärztliche und logopädische Diagnosestellung der Dysphagie sollte erfolgen. Dazu kann auch in der Häuslichkeit und im Pflegeheim über den Hausarzt eine Verordnung ausgestellt werden. Die Pflegepersonen können sich von den Logopäden zur Diagnostik und dem Vorgehen bei Dysphagie beraten lassen.

Pflegerisch besteht die wichtigste Maßnahme der Pneumonieprophylaxe in mindestens zweimal täglicher Mundpflege. Die Mundpflege und das Anreichen von Nahrung dürfen nur nach fachlicher Unterweisung erfolgen. Getränke und Speisen müssen in korrekter aufrechter Sitzposition angeboten werden. Pürierte Kost ist eher nachteilig. Der pürierte Speisebrei verflüssigt sich zu schnell und läuft dann unkontrolliert in den Rachen. Für die Koordination zwischen Speiseaufnahme in den Mund und dem Schlucken hat das Kauen eine wichtige Funktion und sollte nicht durch bereits püriertes Speisenangebot unterbleiben. Beim Essenreichen muss für das Kauen, Einspeicheln und Schlucken ausreichend Zeit eingeplant werden.

\section{Lunge belüften durch Förderung der Mobilität}

Das größte Risiko einer Minderbelüftung der Lunge liegt in der Immobilität. Dazu zählen besonders Bettlägerigkeit, aber auch das lange Verweilen in der immer gleichen Sitzposition oder auch das halbaufrechte Sitzen, wenn die Person aus der Sitzposition in eine halb-liegende Position herunterrutscht. Mobilisation bedeutet, die Person in Bewegung zu bringen. Verfügen die Patienten/Bewohner über ausreichende Bewegungsfähigkeit, sollen sie selbstständig umhergehen, wenn möglich täglich an frischer Luft. Bewegungseingeschränkte Personen sollen mit Unterstützung mehrmals kurze Wege ablaufen. Ist das nicht möglich, muss unbedingt auf eine aufrechte Sitzposition geachtet werden. Außerdem soll das Sitzmöbel im Laufe des Tages mehrmals gewechselt werden. Wann immer möglich, sollen auch massiv bewegungseingeschränkte Personen zeitweilig an die frische Luft gebracht werden.

Atemübungen zur Ein- oder Ausatmung mit entsprechenden Atemtrainern werden empfohlen. Sie können als therapeutisches

\section{† AUf EINEN BLICK: PNEUMONIEN}

Die Lungenentzündung (Pneumonie) ist eine Entzündung des Lungengewebes. Sie wird im Bereich der pflegebedürftigen und alten Menschen zu $90 \%$ durch die Infektion mit Bakterien ausgelöst, seltener durch Viren oder Pilze und durch die Aspiration von Magensaft. Die häufigste Verlaufsform der Pneumonie, die Bronchopneumonie, entsteht meistens durch die Aspiration von Erregern aus dem Nasen-Rachen-Raum oder als bakterielle Superinfektion bei Grippeerkrankung. Die ambulant erworbene Pneumonie ist nach Durchfallerkrankungen weltweit die zweithäufigste Infektionskrankheit. Die Neuinfektion wird allein für Bewohner von Altenheimen auf 68-114 Fälle pro 1.000 Bewohner geschätzt. Das entspricht etwa 400.000600.000 Fällen pro Jahr in Deutschland. Geriater empfehlen, Pneumonie bei alten und pflegebedürftigen Personen immer im Krankenhaus zu behandeln.

Hilfsmittel verordnet werden. Die PEP-Atmung (Positiv Expiratory Pressure) ist eine Ausatmung gegen Widerstand. Der Widerstand wird durch die dosierte Lippenbremse mit nur leicht geöffnetem Lippenspalt erzeugt. Auch hierzu stehen Trainingsgeräte zur Verfügung. Es kann sinnvoll sein, bei hochgefährdeten Patienten/Bewohnern eine physiotherapeutische Beratung und Begleitung verordnen $\mathrm{zu}$ lassen. Physiotherapie lässt sich außerhalb des Krankenhauses auch für pflegebedürftige Personen verordnen (Reha trotz Pflege).

Beschrieben werden spezielle Lagerungen zur Pneumonieprophylaxe für bettlägerige Personen. Die Lagerungen sollen die Lungenbelüftung unterstützen. Für den gesamten Bereich der Lagerungen liegen jedoch keine Wirknachweise vor. Am bekanntesten sind die AVT-Lagerungen. Die Lagerung lässt sich mit den üblichen Kopf- 


\section{+ PNEUMONIEPROPHYLAXE:} MIT LISA PLUS

\author{
$L=$ Lungenbelüftung \\ - Mobilisation \\ - Atemübungen \\ _ Lagerung \\ I = Infektion vermeiden \\ _ $2 x$ täglich Mund- und Nasenpflege \\ - Hygienestandards einhalten \\ $\mathrm{S}=$ Sekretmanagement \\ _ Sekret verflüssigen, lösen und entleeren \\ _ Flüssigkeitsgabe 2 Liter täglich \\ $A=$ Aspiration vermeiden \\ - Oberkörperhochlagerung (OKH) \\ _ Korrektes Sitzen beim Essen- u. Getränkereichen \\ - Normalkost statt passierter Kost \\ - Dauergabe bei Sondenkost \\ plus \\ $M=$ Medizin \\ _ Grippe-Schutzimpfung \\ - Pneumokokken-Impfung \\ - Medikamentenanpassung \\ (Quelle: Häse 2020; Huhn 2020)
}

oder Lagerungskissen nicht herstellen. Es werden schmale und feste Kissen oder Handtücher so angebracht, dass der Brustkorb unterschiedlich gehoben wird. Bei der A-Lagerung ist das der obere Bereich (Schulter), bei der V-Lagerung der untere Bereich (Rippenbogen). Die T-Lagerung mit dem Lagerungsmaterial unter der Wirbelsäule soll eine Dehnung des gesamten Brustkorbs bewirken. So sollen die Lungensegmente entsprechend mehr belüftet werden. Richtig angewendet, erhöht sich bei der AVT-Lagerung der Druck auf Kreuzbein und Wirbelsäule, wodurch das Dekubitusrisiko steigt. Deshalb darf die einzelne Lagerung nicht länger als 20 Minuten durchgeführt werden. Traditionell wird zur Lungenbelüftung die Halbmondlagerung als Dehnlagerung beschrieben. Der Körper wird in eine halbkreisähnliche Position gebracht, wodurch die gegenüberliegende Seite gedehnt wird. Hier soll eine Sogwirkung entstehen, die eine Mehrdurchlüftung bewirkt. Diese Position wird oft als unangenehm erlebt, weshalb sie selten lange eingehalten wird. Dennoch darf keine fixierte Lagerung mittels Kissen vorgenommen werden.

\section{Medizinische Pneumonie-Prophylaxe}

Nationale und internationale Studien verweisen auch in Bezug auf die Prophylaxe der Pneumonie auf die hohe Bedeutung der Grippeschutzimpfung. Zusätzlich wird ab dem 60. Lebensjahr auf eine Pneumokokken-Impfung als Standard mit starker Empfehlung hingewiesen. Selbstverständlich sollte sein, dass die behandelnden Ärzte den Einfluss von Medikamenten auf die Atmung und das Schluckgeschehen berücksichtigen und entsprechend verordnen. Die grundsätzliche Minimierung der Einnahme von Medikamenten und das Vermeiden von Substanzen, die die Speichelproduktion hemmen, reduzieren das Risiko einer Aspirationspneumonie ebenso wie der Verzicht auf sedierende Medikamente. Für verordnete Inhalationen liegt nur ein geringer Wirknachweis zur Pneumonieprophylaxe vor. Sie können jedoch helfen, Sekret im oberen Atmungsbereich zu lösen, was sich symptomlindernd darstellt.

\section{PFLEGE EINFACH MACHEN}

Immobilisierung (Bettlägerigkeit) gilt neben der Aspiration als Hauptrisikofaktor für die im Pflegeheim erworbene Pneumonie.

Nur wenige der beschriebenen Maßnahmen zur Prophylaxe sind jedoch ausreichend belegt. Positiv bewertet werden die Infektionsvermeidung, das Sekretmanagement, Aspirationsvermeidung und medizinische Maßnahmen.

Bei unklaren Symptomen muss die Prüfung der Atemfrequenz (unter 30/Min.) und des Blutdrucks (systolisch unter 90 $\mathrm{mmHg}$, diastolisch unter $60 \mathrm{mmHg}$ ) immer vorgenommen werden.

Auch für die Mobilisation und die Oberkörperhochlagerung gibt es positive Ergebnisse. Belege über die Wirkung und den Nutzen der speziellen Lagerungen liegen nicht vor. Umso wichtiger ist es, Anzeichen einer Pneumonie frühzeitig zu erkennen und die Behandlung rasch einzuleiten.

Schlüsselwörter: Pneumonie, Aspiration, Dysphagie, Immobilität, AVT-Lagerungen

\section{Literatur beim Verfasser}

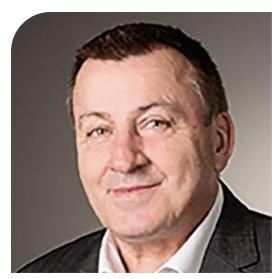

\section{Siegfried Huhn}

Pflegeberatung, Fortbildung, Qualitätsentwicklung Hagelberger Str. 46, 10965 Berlin siegfried.huhn@freenet.de 
Hier steht eine Anzeige.

黛 Springer 